\title{
Microprocessor controller WAGO, as the controller of the active group of radiators in $\mathrm{HV}$ autotransformer
}

\author{
Paweł Kopański, Ryszard Kowalik, Krzysztof Glik
}

\author{
Warsaw University of Technology \\ Institute of Electric Power Engineering \\ Koszykowa 75, Warsaw (Poland) \\ Phone/Fax number: 0048222345608
}

E-mail: pawel.kopanski@ien.pw.edu.pl,ryszard.kowalik@ien.pw.edu.pl,krzysztof.glik@ien.pw.edu.pl

\begin{abstract}
This paper presents the structure and the algorithm of microprocessor controller WAGO, used for the planned control of radiators in transformers of highest power. Apart from that, the requirements concerning the control of radiators will be presented (Microprocessor controller WAGO, as the controller of the active group of radiators in $\mathrm{HV}$ autotransformer).
\end{abstract}

\section{Keywords}

PLC WAGO, Control of radiators, HV autotransformers, microprocessor.

\section{Introduction}

Modern systems which control radiators in transformers and autotransformers of highest power, must realize complex algorithms, which are hard to implement using conventional methods. For this reason, the Institute of Electric Power Engineering at Warsaw University of Technology developed an algorithm that allows for flexible and simple control of radiators. This was achieved using a microprocessor controller WAGO allowing easy implementation of complex logic circuits.

\section{Description of running program}

One of the basic requirements for controller is that following groups of radiators start to operate depending on the actual temperature of windings and oil.

Start of operation should be performed in three steps which indicate that successive temperature thresholds are exceeded. Recommended levels of steps ,1”, „2" and ,3” are shown in table I.

Table I. Parameters of sensor

\begin{tabular}{|c|c|c|c|}
\hline Maximal temperature: & \multicolumn{3}{|c|}{ Step: } \\
\cline { 2 - 4 } &, 1 , &, 2 , &, 3 " \\
\hline Windings: & - & 80 & 100 \\
\hline Oil: & 45 & 55 & 65 \\
\hline
\end{tabular}

Microprocessor controller must also realize the algorithm of switching the leading group of radiators. It aims to change sequence of switching each radiators on. The reason to execute this algorithm is the need to divide the working time of each radiator, so that every radiator is used uniformly.

The change sequence of radiators operation should be performed once a month. Recommended sequence of radiators operation, for transformer with four radiators is shown in the Table. II

Table II. Sequence of radiators operation

\begin{tabular}{|c|c|c|c|}
\hline $\begin{array}{c}\text { Sequence of } \\
\text { radiators } \\
\text { operation }\end{array}$ & \multicolumn{3}{|c|}{ Step: } \\
\cline { 2 - 4 } &, 1, &, $2{ }^{\prime}$ &, $3{ }^{\prime}$ \\
\hline A & 1 & +4 & $+(3+2)$ \\
\hline B & 2 & +1 & $+(4+3)$ \\
\hline C & 3 & +2 & $+(1+4)$ \\
\hline D & 4 & +3 & $+(2+1)$ \\
\hline
\end{tabular}

When the A sequence of radiators operation is chosen, then in first step once the oil temperature is higher than $45^{\circ} \mathrm{C}$, radiator number 1 is switched on. Once the oil temperature is higher than $55^{\circ} \mathrm{C}$, radiator number 4 is also switched on. Once the oil temperature is higher than

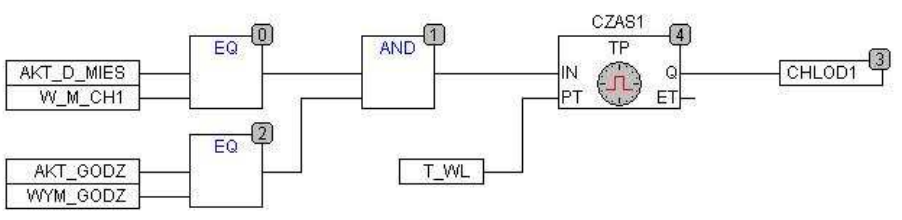

Fig. 1. The algorithm switching radiator on once a month for 10 minutes $65^{\circ} \mathrm{C}$, step " 3 " is started and two remaining radiators (number 3 and 2) are switched on. 


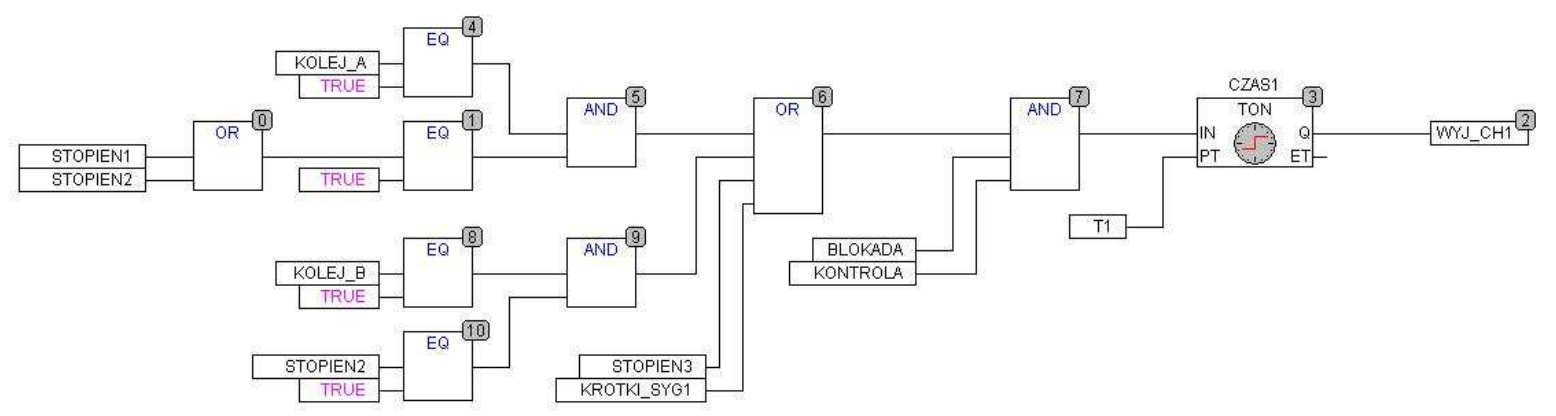

Fig. 2. The algorithm for switching radiator on

In addition, microprocessor controller should switch each radiator to work at least once each month for a short period of time (i.e. 10 minutes), not depending on their work program resulting from the current temperature of autotransformer. In controller it is realized by switching following radiator each week on a specific day and specific time for 10 minutes. The example of realization of this algorithm in program CoDeSys (WAGO programming language) is shown in Figure 1. Information about current day (AKT_D_MIES) and hour (AKT_GODZ) are transmitted to the system, which compares them with set values, which are available through MODBUS IP protocol. If all conditions are met, timer is started, set on 10 minutes and radiators are switched on.

Microprocessor controller must always switch radiators with specified time delay, different for each one of them. This is to prevent the situation where for example, all radiators are switched on at the same time. The controller carries out this function using memory of timer which is assigned to each of radiators with different delay time. The example of realization of switching radiator $\mathrm{nr} 1 \mathrm{on}$ is shown in Figure 2. As it is shown in algorithm, first radiator is switched on in the " 1 " or " 2 " step if the A sequence of radiators operation is active, in step " 2 " for B sequence or in step " 3 " not depending on active sequence. The "BLOKADA" signal is an external signal connected to control system and can block this system. The "KONTROLA" signal comes from the module which switch radiator on once a month for 10 minutes.

All information concerning operating radiator, current leading group and internal time in controller are available by MODBUS IP protocol. This enables to get information about operation of the device using substation supervisory and control system. Additionally, some of the set parameters can be modified i.e. to determine in which month the radiator is set as a leading one.

\section{Architecture of microprocessor controller}

For the purpose of carrying out the functionalities which were described in first part of the article, WAGO controller was equipped with following components: CPU (catalogue nr 750-841), two modules of 2-channel digital inputs (catalogue nr 750-405), five modules of 2channel digital outputs (catalogue nr 750-512) and power supply module(catalogue $\mathrm{nr} 750-612$ ) which enables to connect control signals $220 \mathrm{~V}$ AC and end module (catalogue $\mathrm{nr}$ 750-600). Digital inputs of modules, indicated in Figure 3, as "wej" have the following meaning:

- wej1/1 (first input of first module), operation of the first step of oil temperature sensors in transformer - To $>45^{\circ} \mathrm{C}$,

- wej1/2 (second input of first module), operation of the second step of oil temperature sensors To $>55^{\circ} \mathrm{C}$, or thermal model (windings) $\mathrm{Tu}>80^{\circ} \mathrm{C}$,

- wej2/1 (first input of second module), operation of the third step of oil temperature sensors $\mathrm{To}>65^{\circ} \mathrm{C}$, or second step of thermal model(windings) $-\mathrm{Tu}>100^{\circ} \mathrm{C}$.

- wej2/2 (second input of second module), circuit breaker is closed on the $220 \mathrm{kV}$ side of autotransformer, which causes unblocking the controller.

These inputs use the $\mathrm{AC}$ voltage signal comprising a phase of control voltage signal and a neutral signal. The

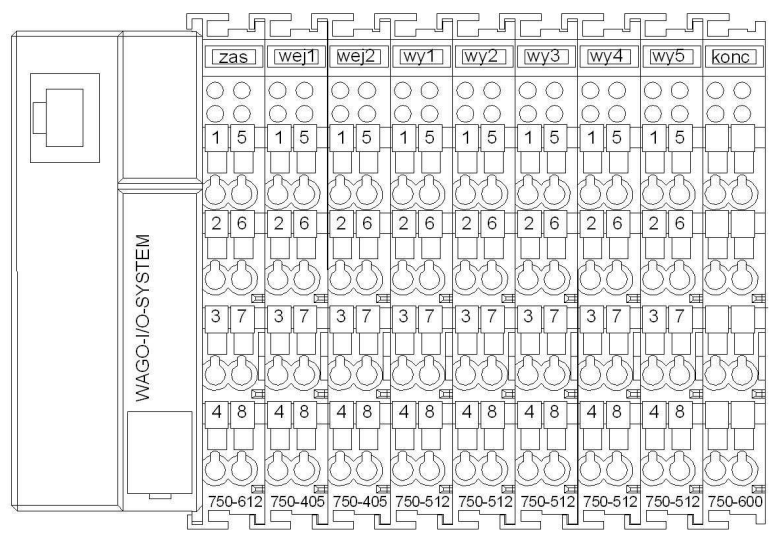

Fig. 3. WAGO controller, which controls oil pumps, radiators and signalizes leading radiator

signal is transmitted to inputs (contacts 1 or 5 of inputs modules in WAGO controller) by devices for measurement of oil temperature and by thermal model of windings.

Neutral signal is fed to the inputs internally in WAGO controller from the contact $\mathrm{nr} 3$ of power supply module.

Binary outputs indicated in Figure 3 as ,wy" have following meaning:

- wy1/1 (first output of first module), switch radiator number 1 on,

- wy1/2 (second output of first module), switch radiator number 2 on, 
- wy2/1 (first output of second module), switch radiator number 3 on,

- wy2/2 (second output of second module), switch radiator number 4 on,

- wy3/1 (first output of third module), leading group of radiators $\mathrm{nr} 1$ (leading radiator $\mathrm{nr} 1$ ),

- wy3/2 (second output of third module), leading group of radiators $\mathrm{nr} 2$,

- wy4/1 (first output of fourth module), leading group of radiators $\mathrm{nr} 3$,

- wy4/2 (second output of fourth module), leading group of radiators $\mathrm{nr} 4$,

- wy5/1 (first output of fifth module), correct work of WAGO controller,

- wy5/2 (second output of fifth module), not used.

These outputs use for signalization the AC signal, which is the phase of control voltage. Power supply module in controller receives the signal through contact $\mathrm{nr} 2$.

Figure 4 shows digital inputs module (catalogue nr 750405) and it's block diagram.

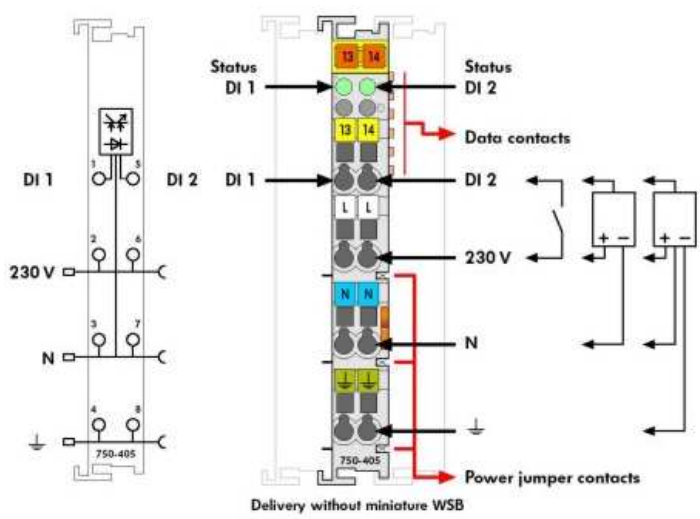

Fig. 4. Digital inputs module (catalogue nr 750-405) WAGO controller

\section{Conclusion}

Application of microprocessor controller, produced by WAGO company, gives almost limitless control options of radiators work. At any time, the implemented program can be changed in order to adapt it to changing needs. Algorithm described above is just one of the proposals. The controller installed in substation realizes algorithm similar to that described in the article. The algorithm is continuously modified and adapted to changing needs. One of the major changes which was made is the change in switching sequence of radiators. To avoid situation when the same group of radiators works in hottest months (June, July), the algorithm was changed, in this way so the switching operation was performed not once a month but once a week.

\section{References}

[1] Project of AT1 transformer monitoring system AT1 in the Poręba substation,

[2] WAGO I/O System 750, ETHERNET TCP/IP 750-841, Manual,

[3] User Manual for PLC Programming with CoDeSys 2.3, S3 - Smart Software Solutions GmbH. 